\title{
A KRONECKER THEOREM FOR HIGHER ORDER HANKEL FORMS
}

\author{
RICHARD ROCHBERG \\ (Communicated by Theodore Gamelin)
}

\begin{abstract}
A classical theorem of Kronecker describes those Hankel forms that are of finite rank. Here an analogous characterization is given for the higher order (higher rank) Hankel forms introduced by Janson and Peetre. The methods apply to spaces of holomorphic functions in which the polynomials are dense.
\end{abstract}

\section{INTRODUCTION AND SUMMARY}

A classical theorem of Kronecker describes the finite-rank Hankel operators or, what is almost the same thing, the finite-rank Hankel forms. In modern language Kronecker's result is for Hankel forms acting on the Hardy space of the unit disk, $D$. See [Po2] for general background. The result can be extended to Hankel forms acting on other spaces ([JPR], [Po1]). Recently the author and others have extended the notion of Hankel bilinear form to introduce Hankel forms of higher order [JP], [PR]. In [PR], a Kronecker style theorem was proved for higher order Hankel forms acting on the Fock space and the question was raised if there might be a general Kronecker theorem for higher order Hankel forms. In fact such a result is true in many contexts and it is the purpose of this note to present that result.

In the next section we recall the definitions of higher order Hankel forms and the basic examples. At the end of that section we state our main result, Theorem 1. The proof of Theorem 1 is in $\S 3$. A brief final section contains some further remarks, including a discussion of a structure theorem for higher order Hankel forms that follows from the proof of Theorem 1 and a mention of some very natural questions that remain open.

\section{Higher ORDER HANKEL FORMS}

Suppose $H$ is a Hilbert space of holomorphic functions on a domain $\Omega \subset$. $\mathrm{C}^{n}$. Let $P_{n}$ be the ring of polynomials on $\mathrm{C}^{n}$. We suppose throughout that $P_{n}$ is contained in and is dense in $H$. Thus our result covers Hankel operators on the Hardy, Bergman, and weighted Bergman spaces on the disk and ball as well as the Fock space. However, the hypothesis excludes some important examples

Received by the editors March 25, 1994.

1991 Mathematics Subject Classification. Primary 47B35.

The author was supported in part by the NSF. 
such as the Bergman space of the half-plane. We return to this point in the final section.

By a bilinear form on $H$ we mean a continuous bilinear map $B$ :

$$
B: H \times H \rightarrow \mathbf{C} \text {. }
$$

$B$ is called a Hankel form if

$$
B(p f, g)-B(f, p g)=0 \quad \forall f, g, p \in P_{n} .
$$

A Hankel form is also called a Hankel form of order 0 . We now proceed inductively. A bilinear form $B$ is called a Hankel form of order $k$ if $\forall p \in P_{n}$, the bilinear form $\delta_{p} B$ defined by

$$
\delta_{p} B(f, g)=B(p f, g)-B(f, p g)
$$

is a Hankel form of order $k-1$. We may also call a form of order $k$ a form of type $k$.

Here are some simple examples. Suppose $n=1$ and $H$ is the Bergman space of the unit disk $D$. Pick $\zeta_{0} \in D$. Here are examples of rank one and rank two Hankel forms of order 0:

$$
B_{1}(f, g)=(f g)\left(\zeta_{0}\right)=f\left(\zeta_{0}\right) g\left(\zeta_{0}\right)
$$

and

$$
B_{2}(f, g)=(f g)^{\prime}\left(\zeta_{0}\right)=f^{\prime}\left(\zeta_{0}\right) g\left(\zeta_{0}\right)+f\left(\zeta_{0}\right) g^{\prime}\left(\zeta_{0}\right) .
$$

Here are examples of rank one and rank two Hankel forms of order 1:

$$
C_{1}(f, g)=B_{1}\left(f^{\prime}, g\right)=f^{\prime}\left(\zeta_{0}\right) g\left(\zeta_{0}\right)
$$

and

$$
C_{2}(f, g)=B_{2}\left(f^{\prime}, g\right)=f^{\prime \prime}\left(\zeta_{0}\right) g\left(\zeta_{0}\right)+f^{\prime}\left(\zeta_{0}\right) g^{\prime}\left(\zeta_{0}\right) .
$$

Roughly, the Kronecker theorem says that all of the finite-rank Hankel forms of order 0 are finite linear combinations of forms such as those in (3) and (4) and their obvious variations. Our result will be, roughly, that the finite-rank Hankel forms of order 1 are linear combinations of the forms in (5) and (6) and their obvious variants.

Here is the precise result.

Theorem 1. Suppose $H$ is a Hilbert space of holomorphic functions defined on $\Omega \subset \mathbf{C}^{n}$. Suppose $P_{n}$ is contained in and is dense in $H$. Suppose $B(\cdot, \cdot)$ is a Hankel form of order $j$ for some $j \geq 0$.

1. If $j=0$, then there are $\alpha_{1}, \ldots, \alpha_{k} \in \mathbf{C}, \zeta_{1}, \ldots, \zeta_{k} \in \Omega$, and constant coefficient partial differential operators $D_{1}, \ldots, D_{k}$ such that $B(\cdot, \cdot)$ is given by

$$
B(f, g)=\sum_{i=1}^{k} \alpha_{i} D_{i}(f g)\left(\zeta_{i}\right) .
$$

2. If $j>0$, then there is a finite-rank Hankel form of order $0, C_{0}$, and there are $n$ finite-rank Hankel forms of order $j-1, C_{1}, \ldots, C_{n}$ so that

$$
B(f, g)=C_{0}(f, g)+\sum_{i=1}^{n} C_{i}\left(\frac{\partial f}{\partial z_{i}}, g\right) .
$$


Notes: 1 . It is elementary to verify the converse is also true.

2. There is a variation of $(8)$ in which the derivatives are on $g$.

3. Let us say that a linear functional, $L$, on $H$, is supported at a single point if there is a $\zeta \in \Omega$ so that for $f \in H, L(f)$ is a linear function of the value of $f$ and (finitely many of) its derivatives at $\zeta$. Thus (7) shows that a finite-rank Hankel form of order 0 is a finite sum of linear functionals supported at single points applied to the product. If we start with this and use (8) inductively, we can get a similar description of the bilinear form in (8). If $B$ is a finite-rank Hankel form of order $j$, then there is a finite set of linear functionals, $\left\{L_{i \alpha}\right\}$, each supported at a single point, so that

$$
B(f, g)=\sum_{i} \sum_{|\alpha| \leq j} L_{i \alpha}\left(\left(\partial^{\alpha} f\right) g\right) .
$$

Here $i$ is an integer index and $\alpha$ is an index that is an $n$-tuple and we are using the standard multi-index notation for partial derivatives.

\section{THE PROOF}

The first statement is Theorem 14.1 of [JPR].

We will do the proof of the second statement in three steps. First we look at $n=j=1$ to show the basic idea. We then do an induction on $j$. We then analyze the case $n>1$ with a reductive algorithm that is, essentially, an induction.

Suppose now $n=j=1$. By our density assumption it is enough to verify (8) for polynomials. By linearity we may assume $f$ is a monomial (of course, we may not allow the forms on the right-hand side of $(8)$ to depend on which monomial). Let $B(\cdot, \cdot)$ be the given finite-rank Hankel form of order 1 . Define the bilinear form $D$ by

$$
D(f, g)=B(z f, g)-B(f, z g)
$$

where we are using $z$ to denote the coordinate function on $\mathbf{C}$. Pick an integer $k>0$. For any polynomial $g$ we have

$$
B\left(z^{k}, g\right)=B\left(z^{k-1}, z g\right)+D\left(z^{k-1}, g\right) .
$$

Using the definition of $D$ again we continue with

$$
B\left(z^{k}, g\right)=B\left(z^{k-2}, z g\right)+D\left(z^{k-2}, z g\right)+D\left(z^{k-1}, g\right) \text {. }
$$

Because $B$ is of order 1 , the form $D$ must be a Hankel form of order 0 and hence

$$
D\left(z^{k-2}, z g\right)=D\left(z^{k-1}, g\right)
$$

and thus

$$
B\left(z^{k}, g\right)=B\left(z^{k-2}, z g\right)+2 D\left(z^{k-1}, g\right) .
$$

The pattern is now clear, and we can continue to

$$
B\left(z^{k}, g\right)=B\left(1, z^{k} g\right)+k D\left(z^{k-1}, g\right),
$$

which we choose to write as

$$
B\left(z^{k}, g\right)=B\left(1, z^{k} g\right)+D\left(\left(z^{k}\right)^{\prime}, g\right) .
$$


Note that this formula also holds when $k=0$. Hence we have it for all monomials and hence for any polynomial $f$

$$
B(f, g)=B(1, f g)+D\left((f)^{\prime}, g\right) \text {. }
$$

This is the required conclusion with

$$
C_{0}(f, g)=B(1, f g) \text { and } C_{1}(f, g)=D(f, g) .
$$

We already noted $D$ was a Hankel form of order $0, C_{0}$ is a Hankel form of order zero because it only depends on the product of its arguments. Finally, the fact that $B$ is finite rank ensures that $D$ and $C_{0}$ are both finite rank. Hence we have the required representation.

We stay with functions of a single variable and proceed by induction on $j$. Suppose that we have established the representation (9) for forms of order $j$ or less and we are given a form $B(\cdot, \cdot)$ of order $j+1$. Define $D$ as before. $D$ will be a form of order $j$, and hence by the induction hypothesis and (9) we have a representation

$$
D(f, g)=\sum_{i=0}^{j} L_{i}\left(f^{(i)} g\right) .
$$

We start as before and get

$$
B\left(z^{k}, g\right)=B\left(z^{k-1}, z g\right)+\sum L_{i}\left(\left(z^{k-1}\right)^{(i)} g\right)
$$

and continue with

$$
B\left(z^{k}, g\right)=B\left(z^{k-2}, z^{2} g\right)+\sum L_{i}\left(\left(z^{k-2}\right)^{(i)} z g\right)+\sum L_{i}\left(\left(z^{k-1}\right)^{(i)} g\right),
$$

which we rewrite as

$$
B\left(z^{k}, g\right)=B\left(z^{k-2}, z^{2} g\right)+\sum L_{i}\left(\left(\left(z^{k-2}\right)^{(i)} z+\left(z^{k-1}\right)^{(i)}\right) g\right) .
$$

If we continue in this way we obtain

$$
B\left(z^{k}, g\right)=B\left(1, z^{k} g\right)+\sum L_{i}\left(c_{k, i} z^{k-i-1} g\right),
$$

where

$$
c_{k, i}=\sum_{r=i}^{k-1} r(r-i) \cdots(r-i+1) .
$$

Claim. $c_{k, i}=(i+1)^{-1} k(k-1)(k-2) \cdots(k-i)$.

Once we have the claim we can finish as before. We will have

$$
B\left(z^{k}, g\right)=B\left(1, z^{k} g\right)+\sum L_{i}\left((i+1)^{-1}\left(z^{k}\right)^{(i+1)} g\right) .
$$

Thus, absorbing the numerical constant into the definition of the $L$ 's we have

$$
B(f, g)=B(1, f g)+\sum_{i=0}^{j} L_{i}\left((f)^{(i+1)} g\right),
$$

which is the required version of (9) for order $j+1$. To establish the claim set $d_{k, i}=(i+1)^{-1} k(k-1)(k-2) \cdots(k-i)$, and note that the $c$ 's and the $d$ 's satisfy the same difference equation and agree at one spot and, hence, at all spots. In particular,

$$
c_{k, i}-c_{k-1, i}=d_{k, i}-d_{k-1, i} \text { and } c_{i+1, i}=d_{i+1, i}=i ! .
$$


We now go to the case of several variables. We will actually suppose $\Omega \subset \mathbf{C}^{2}$ with coordinates $z, w$ but it will be clear that the argument is general. We proceed by induction on $j$, the order. We have the case $j=0$ from the first part of the theorem. (We only did the case $n=j=1$ explicitly for the reader's convenience. We could have also started the previous induction from $j=0$.) Suppose we have established (9) for forms of order at most $j$ and we are given a form $B(\cdot, \cdot)$ of order $j+1$. Define $D$ by $D(f, g)=B(f w, g)-B(f, g w)$. Set

$$
\partial_{1}=\frac{\partial}{\partial z} \text { and } \partial_{2}=\frac{\partial}{\partial w} .
$$

The induction hypothesis ensures that $D$ is a Hankel form of order $j$ and hence, by (9),

$$
D(f, g)=\sum_{i} \sum_{\alpha_{1}+\alpha_{2} \leq j} L_{i \alpha}\left(\left(\partial_{1}^{\alpha_{1}} \partial_{2}^{\alpha_{2}} f\right) g\right) .
$$

Here $\alpha=\alpha_{1}+\alpha_{2}$. For any monomial $z^{s} w^{k}$ we have

$$
B\left(z^{s} w^{k}, g\right)=B\left(z^{s} w^{k-1}, w g\right)+\sum_{i} \sum_{\alpha_{1}+\alpha_{2} \leq j} L_{i \alpha}\left(\left(\partial_{1}^{\alpha_{1}} \partial_{2}^{\alpha_{2}} z^{s} w^{k-1}\right) g\right) .
$$

We continue as before and eventually obtain

$$
B\left(z^{s} w^{k}, g\right)=B\left(z^{s}, w^{k} g\right)+\sum_{i} \sum_{\alpha_{1}+\alpha_{2} \leq j} L_{i \alpha}\left(\left(\partial_{1}^{\alpha_{1}} z^{s} c_{k, \alpha_{2}} w^{k-\alpha_{2}-1}\right) g\right) .
$$

As before we rewrite this, absorbing a constant factor into the definition of the $L$ 's, as

$$
B\left(z^{s} w^{k}, g\right)=B\left(z^{s}, w^{k} g\right)+\sum_{i} \sum_{\alpha_{1}+\alpha_{2} \leq j} L_{i \alpha}\left(\left(\partial_{1}^{\alpha_{1}} \partial_{2}^{\alpha_{2}+1} z^{s} w^{k}\right) g\right) .
$$

Now we analyze the first term on the right. $H$ defined by

$$
H(f, g)=B(z f, g)-B(f, z g)
$$

is a form of order $j$, and we can use (9) and the induction hypothesis to write $H$ in terms of functionals $L_{i \alpha}^{\prime}$. We repeat the previous analysis on this form and find that

$$
B\left(z^{s}, w^{k} g\right)=B\left(1, z^{s} w^{k} g\right)+\sum_{i} \sum_{\alpha_{1}+\alpha_{2} \leq j} L_{i \alpha}^{\prime}\left(\left(\partial_{1}^{\alpha_{1}+1} z^{s}\right) w^{k} g\right) .
$$

(The first argument in $B$ does not involve the second coordinate, hence any $L$ that involves $\partial_{2}$ will give a contribution of 0 .) Combining everything and noting that

$$
\left(\partial_{1}^{\alpha_{1}+1} z^{s}\right) w^{k} g=\left(\partial_{1}^{\alpha_{1}+1} z^{s} w^{k}\right) g
$$

we find

$$
\begin{aligned}
B\left(z^{s} w^{k}, g\right)= & B\left(1, z^{s} w^{k} g\right)+\sum_{i} \sum_{\alpha_{1}+\alpha_{2} \leq j} L_{i \alpha}\left(\left(\partial_{1}^{\alpha_{1}} \partial_{2}^{\alpha_{2}+1} z^{s} w^{k}\right) g\right) \\
& +\sum_{i} \sum_{\alpha_{1}+\alpha_{2} \leq j} L_{i \alpha}^{\prime}\left(\left(\partial_{1}^{\alpha_{1}+1} z^{s} w^{k}\right) g\right) .
\end{aligned}
$$


This derivation assumes $s, k>0$, however the formula continues to hold if this fails. We have the formula for all monomials, and hence, for any polynomial $f$

$$
\begin{aligned}
B(f, g)= & B(1, f g)+\sum_{i} \sum_{\alpha_{1}+\alpha_{2} \leq j} L_{i \alpha}\left(\left(\partial_{1}^{\alpha_{1}} \partial_{2}^{\alpha_{2}+1} f\right) g\right) \\
& +\sum_{i} \sum_{\alpha_{1}+\alpha_{2} \leq j} L_{i \alpha}^{\prime}\left(\left(\partial_{1}^{\alpha_{1}+1} f\right) g\right) .
\end{aligned}
$$

This is (9) for forms of order $j+1$. We are done.

\section{NOTES AND COMMENTS}

1. The hypothesis that the polynomials be dense in $H$ seems unfortunate. Certainly it is used in the proof. It is easy enough to modify the proof to cover slightly more general situations such as the Bergman space of an annulus centered at the origin. It is not clear, however, how this type of proof could be extended to, say, a space of functions on a nonplanar Riemann surface. In fact, it is not even clear what happens on the Hardy or Bergman space of the upper half-plane.

2. The theorem proved is actually a theorem about bilinear forms on polynomial rings which satisfy certain additional conditions. It may be that there is a proof of this result, and possibly a more general one, which is more in the spirit of abstract algebra and less computational. The proof of the first part of the theorem given in [JPR] is cast in the language of the ideal theory of polynomial rings. It may be that there is an analogous proof of the second part.

3 . The proof actually produces a structure theorem for various classes of Hankel operators. Suppose $B$ is a Hankel form of order $j$ which is bounded. Suppose that $\Omega \subset \mathbf{C}^{n}$ is bounded. Then the forms such as $H_{i}$ defined by

$$
H_{i}(f, g)=B\left(z_{i} f, g\right)-B\left(f, z_{i} g\right)
$$

is bounded. (This uses the boundedness of $\Omega$.) The proof then gives a representation of $B$ of the sort given in (8) and (9). Now, however, the $C$ 's and the $L$ 's will be bounded. A similar comment applies to compact forms and forms in the Schatten-von Neumman ideals. However, it is not clear if there is a good converse statement. If they have a representation of the sort given in (8) and the $C$ 's are bounded, there is no reason why $B$ should be.

\section{REFERENCES}

[JP] S. Janson and J. Peetre, A new generalization of Hankel operators (the case of higher weight), Math. Nachr. 132 (1987), 318-328.

[JPR] S. Janson, J. Peetre, and R. Rochberg, Hankel forms and the Fock space, Rev. Mat. Iberoamericana 3 (1987), 61-138.

[PR] J. Peetre and R. Rochberg, Higher order Hankel forms, Proc. Conf. Operator Theory (Seattle, 1993) (to appear).

[Po1] S. C. Power, Finite rank multivariable Hankel forms, Linear Algebra Appl. 48 (1984), 237-244.

[Po2] _ Hankel operators on Hilbert space, Pitman, New York, 1982.

Department of Mathematics, Washington University, St. Louis, Missouri 63130

E-mail address: rr@math. wustl. edu 